\title{
Neuropharmacological and Neurobiological Relevance of In Vivo 'H-MRS of GABA and Glutamate for Preclinical Drug Discovery in Mental Disorders
}

\author{
Conny F Waschkies',2, Andreas Bruns', Stephan Müller ${ }^{3}$, Martin Kapps ${ }^{4}$, Edilio Borroni', \\ Markus von Kienlin', Markus Rudin ${ }^{2,5}$ and Basil Künnecke*,I \\ 'pRED, Pharma Research \& Early Development, DTA Neuroscience, F. Hoffmann-La Roche, Basel, Switzerland; nstitute for Biomedical \\ Engineering, ETH and University of Zürich, Zürich, Switzerland; ${ }^{3}$ pRED, Pharma Research \& Early Development, Discovery Technologies, \\ F. Hoffmann-La Roche, Basel, Switzerland; ${ }^{4}$ pRED, Pharma Research \& Early Development, DMPK and Bioanalytical R\&D, F. Hoffmann-La \\ Roche, Basel, Switzerland; ${ }^{5}$ Institute of Pharmacology and Toxicology, University of Zürich, Zürich, Switzerland
}

\begin{abstract}
Proton magnetic resonance spectroscopy ( ${ }^{\text {H }} \mathrm{H}$-magnetic resonance spectroscopy (MRS)) is a translational modality with great appeal for neuroscience since the two major excitatory and inhibitory neurotransmitters, glutamate, and GABA, can be noninvasively quantified in vivo and have served to explore disease state and effects of drug treatment. Yet, if ' H-MRS shall serve for decision making in preclinical pharmaceutical drug discovery, it has to meet stringent requirements. In particular, 'H-MRS needs to reliably report neurobiologically relevant but rather small changes in neurometabolite levels upon pharmacological interventions and to faithfully appraise target engagement in the associated molecular pathways at pharmacologically relevant doses. Here, we thoroughly addressed these matters with a three-pronged approach. Firstly, we determined the sensitivity and reproducibility of ' $\mathrm{H}-\mathrm{MRS}$ in rat at 9.4 Tesla for detecting changes in GABA and glutamate levels in the striatum and the prefrontal cortex, respectively. Secondly, we evaluated the neuropharmacological and neurobiological relevance of the MRS readouts by pharmacological interventions with five well-characterized drugs (vigabatrin, 3-mercaptopropionate, tiagabine, methionine sulfoximine, and riluzole), which target key nodes in GABAergic and glutamatergic neurotransmission. Finally, we corroborated the MRS findings with ex vivo biochemical analyses of drug exposure and neurometabolite concentrations. For all five interventions tested, 'H-MRS provided distinct drug dose-effect relationships in GABA and glutamate over preclinically relevant dose ranges and changes as low as $6 \%$ in glutamate and 12\% in GABA were reliably detected from $16 \mathrm{~mm}^{3}$ volumes-of-interest. Taken together, these findings demonstrate the value and limitation of quantitative ${ }^{1} \mathrm{H}-\mathrm{MRS}$ of glutamate and GABA for preclinical pharmaceutical research in mental disorders.

Neuropsychopharmacology (20I4) 39, 233 I-2339; doi: I0.I038/npp.20 I4.79; published online I4 May 2014
\end{abstract}

\section{INTRODUCTION}

In vivo ${ }^{1} \mathrm{H}$-magnetic resonance spectroscopy (MRS) has been put forward as a unique translational tool for noninvasively monitoring neurobiological alterations in mental disorders and neuropharmacological effects of treatment in man and animal models. Dysfunction of glutamatergic and GABAergic neurotransmission with accompanying excitatory-inhibitory imbalance has been identified as a major substrate of numerous mental disorders (Carlsson et al, 2001; Sanacora and Saricicek, 2007) and novel strategies for pharmacological treatments aim at rectifying the respective neural circuitries (Hashimoto et al, 2004; Krystal et al, 2002;

\footnotetext{
*Correspondence: Dr B Künnecke, Magnetic Resonance Imaging \& Spectroscopy, F. Hoffmann-La Roche, PCDDF, Building 68/327A, Grenzacherstrasse 124, Basel CH-4070, Switzerland, Tel: +4I 61688 2597, Fax: +4I 61687 1910, E-mail: basil.kuennecke@roche.com Received 6 December 2013; revised 28 March 2014; accepted 31 March 2014; accepted article preview online 3 April 2014
}

Moghaddam and Javitt, 2012). In this respect, in vivo MRS has great appeal in that numerous clinical and preclinical studies have demonstrated that the major excitatory and inhibitory neurotransmitters glutamate and GABA are readily amenable by ${ }^{1} \mathrm{H}$-MRS and thus could potentially serve as biomarkers for disease state and treatment efficacy (for reviews see: (Lee et al, 2012; Mattson et al, 1998)). Yet, the value of such in vivo ${ }^{1} \mathrm{H}-\mathrm{MRS}$ readouts as quantitative tools for decision making in preclinical drug discovery in neurology remains to be further established.

In the field of mental disorders, the primary challenges for pharmacological in vivo ${ }^{1} \mathrm{H}$-MRS (subsequently termed 'pharmacoMRS') of neurometabolites implicated in GABAergic and glutamatergic neurotransmission are to reliably assess neurobiologically relevant but rather small changes in respective neurometabolite levels elicited upon pharmacological interventions and to faithfully report target engagement in the associated molecular pathways at pharmacologically relevant doses. PharmacoMRS is thought to report bulk metabolite levels at a macroscopic spatial 
resolution. Yet, although present at millimolar concentrations in the central nervous system, the pools of glutamate and GABA and their precursor glutamine are compartmentalized and neurotransmission is primarily driven by mere relocation of neurotransmitters among these compartments. To date, only few preclinical pharmacoMRS studies with focus on these latter neurometabolites have been conducted in the domains of mental disorders. For example, the aggregate glutamate-glutamine pool in rat brain was shown to be increased by the vigilance-enhancing drug modafinil (Pierard et al, 1995). Likewise, a pronounced increase in the glutamine/glutamate ratio was reported in the frontal cortex of rats after single administration of the psychotomimetic phencyclidine (Iltis et al, 2009). Recently, antidepressant treatment with despiramine was shown to revert the forced swim test-induced increase in cerebral glutamate in mice (Kim et al, 2010). Furthermore, increased cerebral GABA levels were reported in several studies on rats treated with the anti-epileptic vigabatrin, eg (de Graaf et al, 2006; Preece et al, 1994). Although these studies suggest that the fundamental notion of pharmacoMRS of glutamate and GABA is viable, a systematic and comprehensive assessment of sensitivity and reliability of the readouts and their neuropharmacological and neurobiological relevance is pending.

Here, we set out to complement previous work by comprehensively evaluating the merits and limitations of pharmacoMRS as a standard tool for preclinical drug discovery in mental disorders. For this purpose we adopted a three-pronged approach. Firstly, we determined the generic sensitivity and reproducibility of pharmacoMRS at 9.4 $\mathrm{T}$ for detecting changes in neurometabolite levels in two neurobiologically distinct regions of rat brain, ie the striatum and the prefrontal cortex as representatives with strong GABAergic and glutamatergic innervation, respectively. Secondly, the pharmacological and neurobiological relevance of pharmacoMRS readouts was evaluated in these regions using five well-characterized pharmacological interventions that specifically modulate key nodes of GABAergic and glutamatergic neurotransmission, respectively. Drug dose-effect relations over preclinically relevant dose ranges were established to assess the fidelity of pharmacoMRS in reporting mechanism-driven alterations in GABA, glutamate, and glutamine levels. Finally, pharmacoMRS findings were further validated with ex vivo biochemical analyses of drug exposure and cerebral concentrations of GABA and glutamate.

\section{MATERIALS AND METHODS}

\section{Animals and Pharmacological Interventions}

All procedures with animals were conducted in strict adherence to the Swiss federal ordinance on animal protection and welfare.

PharmacoMRS assessments were carried out within a period of 15 months on five cohorts of male SpragueDawley rats (Charles River Laboratories, France) weighing $340 \mathrm{~g}+/-30 \mathrm{~g}$. In a vehicle-controlled parallel design, each cohort (referred to as 'study arm' in the following) was subjected to an acute-pharmacological intervention with either vigabatrin $(30,100$, and $300 \mathrm{mg} / \mathrm{kg}$ intraperitoneally (i.p.)), tiagabine (10, 20, and $40 \mathrm{mg} / \mathrm{kg}$ per os), 3-mercap- topropionate (3-MP; 20, 30, and $40 \mathrm{mg} / \mathrm{kg}$ i.p.), riluzole (3, 6, and $12 \mathrm{mg} / \mathrm{kg}$ i.p ), or L-methionine sulfoximine (MSO; 50, 100, and $200 \mathrm{mg} / \mathrm{kg}$ i.p.). All conditions were tested with 6-8 animals per dose group.

For pharmacoMRS, animals were maintained under anesthesia (1.8-2.4\% isoflurane in 1:5 oxygen:air mixture supplied by face mask) and rectal temperature, respiration rate and end-tidal $\mathrm{CO}_{2}$ monitored, and tightly regulated. The total duration of pharmacoMRS including animal preparation was $60 \mathrm{~min}$. After completion of the pharmacoMRS measurements, animals were killed by decapitation and tissues sampled for further ex vivo analyses.

\section{In vivo ${ }^{1} \mathrm{H}$ pharmacoMRS and MRI}

${ }^{1} \mathrm{H}$ PharmacoMRS was carried out on a Biospec $9.4 \mathrm{~T} / 20 \mathrm{~cm}$ animal scanner (Bruker, Ettlingen, Germany) equipped with a volume resonator for excitation and a surface coil for signal reception. Two regions of interest of $16 \mathrm{~mm}^{3}$ volume in the right striatum and the medial prefrontal cortex, respectively, were investigated. Local magnetic field homogeneity was adjusted and pharmacoMRS was performed with point-resolved spectroscopy at TR/TE $=2000 \mathrm{~ms} / 10 \mathrm{~ms}$, $4 \mathrm{kHz}$ spectral width, 2048 data points and 512 averages collected over a total acquisition time of $17 \mathrm{~min}$ with outer volume and water suppression (a more detailed description is available in the Supplementary Material). Quantitative and unbiased evaluation of the spectral data was performed with LCModel (Provencher, 1993) integrated into a fully automated analysis pipeline.

\section{Ex vivo Quantification of GABA and Glutamate Levels and Drug Exposure}

Tissue levels of GABA and glutamate were quantified by means of isotope dilution measured with liquid chromatography-tandem mass spectrometry (LC-MS/MS). Tissue extracts were prepared from biopsies of the striatum and the prefrontal cortex and spiked with an isotope standard before injection into the LC-MS/MS. The analytes were eluted from the column with a binary mobile phase gradient and the specific transitions associated with the mass-to-charge ratios $m / z=104-87, m / z=148-84$, and $m / z=110-93$ were monitored for the quantification of GABA, glutamate, and the isotope standard $\left(2,2,3,3,4,4-\mathrm{D}_{6}\right)-\mathrm{GABA}$, respectively.

Drug exposures were assessed by LC-MS/MS in extracts prepared from plasma and brain tissue. Respective drugs were eluted with binary mobile phase gradients and detected by monitoring single transitions from parent to specific fragments. Drug quantification was accomplished by referencing to a previously established calibration curve.

\section{Statistical Analyses}

Statistical analyses were performed using JMP 8.0.1 (SAS Institute). The spectra of two animals were excluded from analysis as a consequence of inappropriate drug dosing and highly aberrant neural glutamine metabolism, respectively. A variance components analysis based on the vehicle-treated animals of all five study arms was conducted to appraise between-subject variability of metabolite concentrations and between-group reproducibility. Unbounded variance 
estimates of the study arm factor served as measure of between-group reproducibility in this analysis with study arm and animal (nested within study arm) as random effects.

Brain region-specific differences and changes in neurometabolite concentrations upon drug treatment were assessed separately for GABA, glutamate and glutamine by two-way ANOVAs with treatment as grouping factor and brain region as within-subject factor. Post-hoc $t$-tests were used to compare single drug doses to vehicle, whereas doseeffect relations were assessed by linear-trend tests. To provide most generic data, significances are given uncorrected for multiple testing except where indicated otherwise. Data are presented as mean \pm standard error of the mean.

\section{RESULTS}

\section{Quality, Reliability and Sensitivity of In vivo ${ }^{1} \mathrm{H}$ pharmacoMRS}

Quantitative in vivo ${ }^{1} \mathrm{H}$ pharmacoMRS in the striatum and the prefrontal cortex was performed under conditions of routine operation over five study arms comprising 155 rats and resulted in more than 300 individual spectra that were evaluated. All spectra were of high quality as is exemplified in Figure 1 with representative data obtained from individual animals that received ascending doses of vigabatrin. The well-described resonances of cerebral metabolites including glutamate, glutamine, and GABA are clearly discerned. Spectral analysis with LCmodel provided quantitative appraisals of metabolite concentrations, Cramer-Rao lower bounds (CRLBs) as an estimate for the accuracy of their quantification, and measures of spectral quality. An average overall signal-to-noise (SNR) ratio of 11.5 and 15.3, and an average line width of the water resonance of 10.1 and $8.7 \mathrm{~Hz}$ were routinely achieved in the striatum and the prefrontal cortex, respectively. The inset in Figure 1 tangibly supports the excellent spectral resolution: the resonances of phosphocreatine $(\mathrm{PCr})$ and creatine $(\mathrm{Cr})$, which lie merely $0.017 \mathrm{ppm}(6.8 \mathrm{~Hz}$ at $9.4 \mathrm{~T})$ apart, were regularly resolved as partially separated peaks. In all spectra, CRLBs for glutamate, glutamine and GABA were well below the usual goodness-of-fit exclusion criterion of $20 \%$ and average CRLBs were lower than 3\% (upper 95th percentile, $4 \%$ ), $8 \%$ (upper 95 th percentile, $10 \%$ ), and $9 \%$ (upper 95th percentile 12\%), respectively.

A variance components analysis on the pooled data of the vehicle groups in all study arms delivered estimates for the biological and technical variability of metabolite quantification as shown in Figure 2 for GABA, glutamate, and glutamine. Advantage was taken of the study's outline in that data acquisition for each study arm was completed within 5 days whereas the five study arms were spread over a period of 15 months. Between-group variability of the vehicle-treated animals across study arms was not significantly different from zero (ie, the lower $95 \%$ confidence limit was negative), thus indicating excellent long-term stability. Between-subject variability, as a measure of biological variability in the population, was below $4.5,8$ and $11 \%$ for glutamate and GABA, respectively. Prospective statistical power calculations project that minimal changes of $6 \%$ in glutamate, $12 \%$ in GABA, and $16 \%$ in glutamine

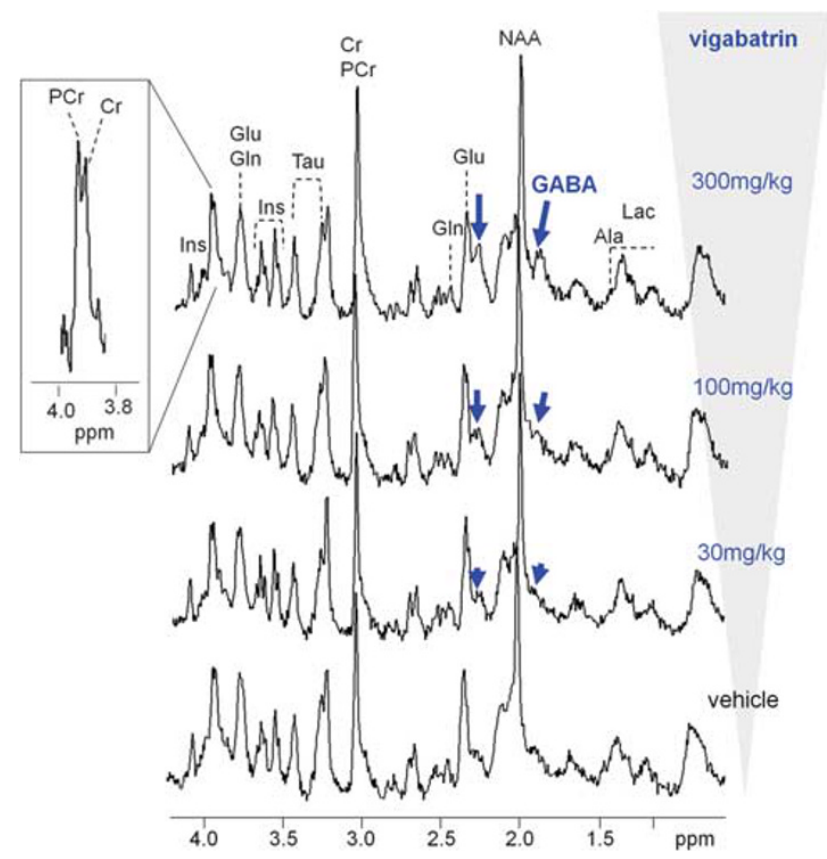

Figure I Representative in vivo ' $\mathrm{H}$ pharmacoMRS spectra of rat prefrontal cortex obtained under routine conditions at 9.4T. Spectra were recorded over $17 \mathrm{~min}$ from $16 \mathrm{~mm}^{3}$ volumes-of-interest in individual animals upon acute-pharmacological intervention with different doses of vigabatrin. High-spectral quality (inset demonstrates spectral resolution by separated peaks of the phosphocreatine and creatine resonances) and reproducibility was attained, allowing a drug dose-related change in GABA levels (arrows) to be observed. Data were processed with IHz exponential line broadening (main display) or Gaussian broadening (inset). Ala, alanine; $\mathrm{Cr}$, creatine; GABA, gamma-aminobutyric acid; Glu, glutamate; Gln, glutamine; Ins, myo-inositol; Lac, lactate; NAA, N-acetylaspartate; PCr, phosphocreatine; Tau, taurine.

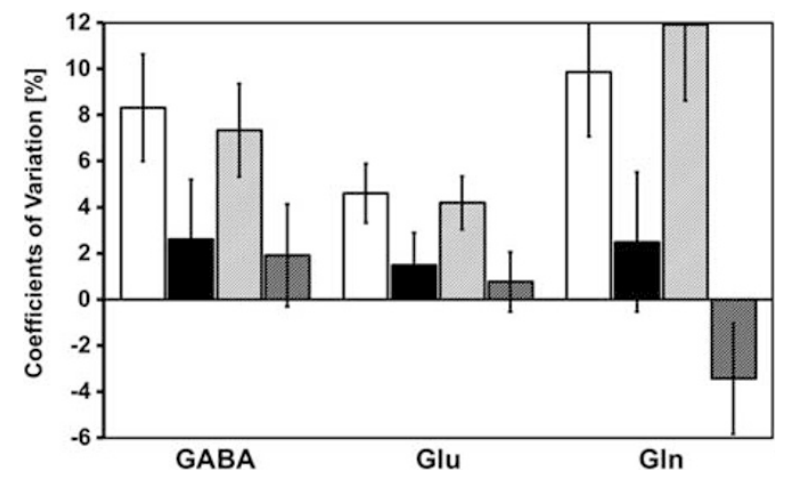

Figure 2 Variance components analysis on GABA, glutamate, and glutamine concentrations detected by in vivo ' $\mathrm{H}$ pharmacoMRS in rat striatum (solid bars) and prefrontal cortex (hatched bars) of all vehicletreated animals. Data were collected in five study arms distributed over 15 months. Each study arm was completed within 5 days. Between-group variability (dark bars) was not significantly larger than zero, hence indicating excellent long-term stability. Between-subject variability (light bars) depicts biological variability in the population. Error bars indicate $95 \%$ confidence intervals.

can be detected with a power of $80 \%, \alpha=5 \%$, and a group size of $n=8$ animals (plain parallel-group design with unpaired $t$-test). 
In vivo ${ }^{1} \mathrm{H}$ pharmacoMRS Detects Pharmacological Modulations of GABA and Glutamate

Fidelity and sensitivity of pharmacoMRS for reporting changes in neurometabolite levels in response to pharmacological interventions was assessed by specifically modulating key nodes of GABAergic and glutamatergic neurotransmission with five well-characterized pharmacological agents as schematically depicted in Figure 3. Accordingly, drug dose-effect relations over preclinically relevant dose ranges were established for GABA, glutamate, and glutamine by means of pharmacoMRS in the striatum and the medial prefrontal cortex. Figure 4 summarizes the quantitative outcomes.

First, GABA catabolism was inhibited with vigabatrin, a clinically used irreversible inhibitor of the GABA-transaminase (GABA-T). A strong dose-dependent increase $(p<0.001$, corrected for multiple testing) in GABA was observed both for the striatum and the prefrontal cortex and reached a maximum of 50 and $110 \%$ over baseline (vehicle), respectively (c.f. Figure 1 for raw data). For the intermediate dose of $100 \mathrm{mg} / \mathrm{kg}$ of vigabatrin an unambiguous increase in GABA of $17 \%(p<0.05)$ was detected in the prefrontal cortex, whereas in the striatum the effect was $12 \%$ and missed significance $(p=0.12)$. In addition to the repercussions on GABA, vigabatrin also had distinct downstream effects that revealed a significant drop in glutamate level by $5 \%$ in the prefrontal cortex at a dose of $300 \mathrm{mg} / \mathrm{kg}$ vigabatrin, and a dose-independent increase in the striatal glutamine of $10-17 \%$ that reached significance $(p<0.05)$ at the doses of $30 \mathrm{mg} / \mathrm{kg}$ and $300 \mathrm{mg} / \mathrm{kg}$, respectively.

In the second study arm, GABA synthesis was blocked at the glutamic acid decarboxylase (GAD) with the competitive inhibitor 3-MP. Accordingly, 3-MP dose-dependently decreased GABA in the striatum $(p<0.05$; corrected for multiple testing). Notably, doses of 30 and $40 \mathrm{mg} / \mathrm{kg} 3-\mathrm{MP}$ resulted in significant reductions of GABA by $8 \%(p<0.05)$ and $12 \%(p<0.01)$, respectively. GABA in the prefrontal cortex, however, was unaffected and remained at baseline levels. In contrast, 3-MP had an indirect effect on glutamate in the prefrontal cortex and at doses of 20 and $30 \mathrm{mg} / \mathrm{kg}$ led to a small but significant drop of 5\% $(p<0.05)$ from baseline level. Glutamine levels remained unchanged upon intervention with 3-MP.

Thirdly, upon treatment with the clinically used selective GABA transporter (GAT) subtype 1 inhibitor tiagabine GABA levels in the striatum were found to be consistently but not dose-dependently elevated by $9 \%(p<0.05), 7$ and $8 \%(p<0.14)$, whereas in the prefrontal cortex no change was observed. Yet, the pharmacoMRS data suggest a dosedependent decrease in glutamate. This secondary effect was more pronounced $(-5 \%)$ in the prefrontal cortex and reached statistical significance $(p<0.05)$ at the mid and higher doses of tiagabine. Furthermore, a significant ( $p<0.01$, corrected for multiple testing) dose-dependent increase in glutamine was observed in the striatum $(18 \%$ at the dose of $80 \mathrm{mg} / \mathrm{kg}$ tiagabine, $p<0.01$ ), but not in the prefrontal cortex.

Analogous to the specific modulations of the GABAergic system, glutamatergic neurotransmission was targeted pharmacologically in two more arms of the study. Recycling of synaptically released glutamate in the glial-neuronal glutamate-glutamine cycle was blocked with the irreversible glutamine synthetase (GS) inhibitor MSO. Glutamate was found to be reduced significantly and dose-dependently in the prefrontal cortex $(p<0.01$, corrected for multiple testing), whereas in the striatum the effect barely missed significance $(p<0.06$, corrected for multiple testing). At all doses tested, MSO lead to a significant $(p<0.05)$ drop in glutamate of $\sim 8 \%$ and more. In addition, the highest dose of MSO also had repercussions on the GABAergic system and led to a significant reduction in the striatal GABA by $9 \%$ from baseline $(p<0.05)$ and a substantial increase in

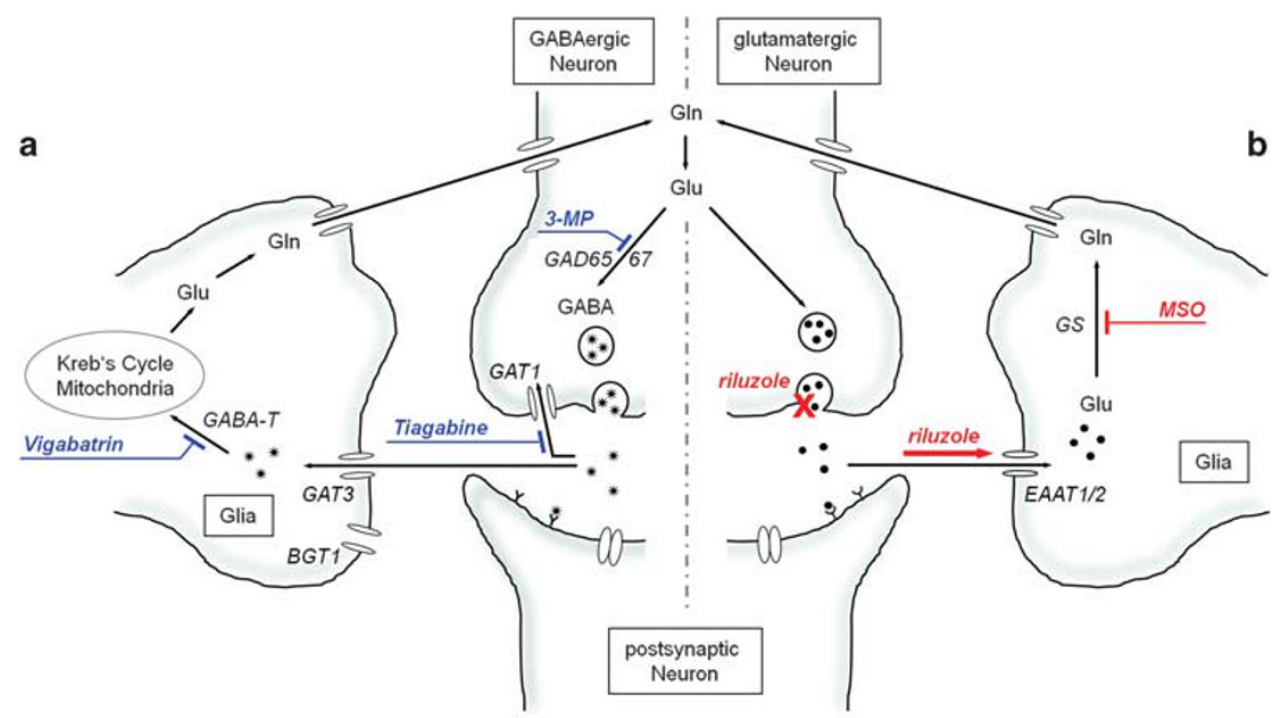

Figure 3 Schematic view of GABAergic (a) and glutamatergic (b) neurotransmission and neurometabolism, and their targeting by specific pharmacological interventions. Pharmacological relevance of pharmacoMRS was ascertained by modulating key nodes of the respective neurotransmitter system with the prototypical GABAergic agents vigabatrin, tiagabine and 3-mercaptopropionate, and the glutamatergic agents riluzole and L-methionine sulfoximine. BGTI, betaine-GABA transporter I; EAATI/2, excitatory amino acid transporter I/2; GABA-T, GABA-transaminase; GAD, glutamate decarboxylase; GATI, GABA transporter I; GS, glutamine synthetase; MSO, L-methionine sulfoximine; 3-MP, 3-mercaptopropionate. 

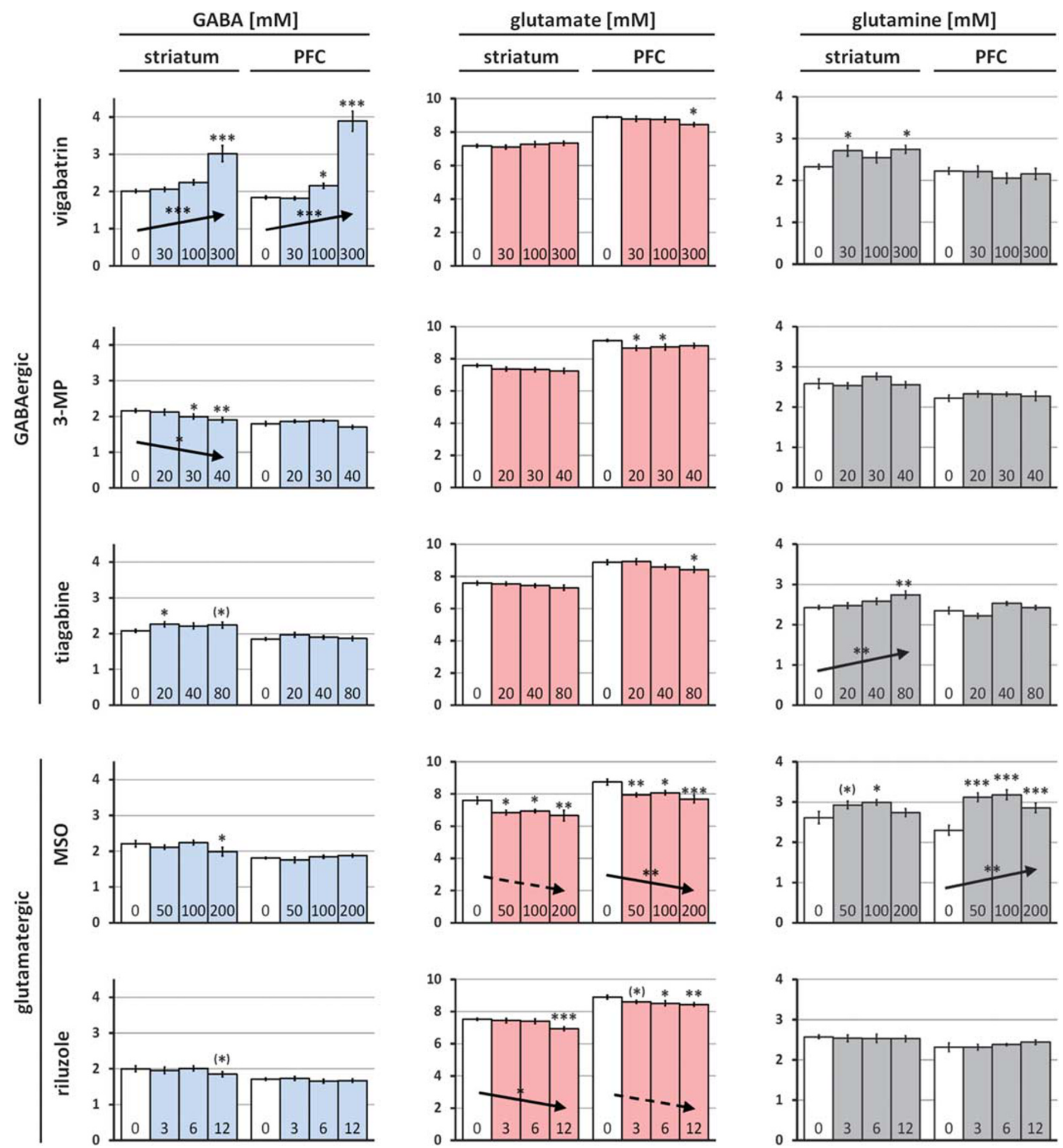

Figure 4 Modulation of cerebral GABA, glutamate, and glutamine levels as detected by in vivo 'H pharmacoMRS in rat striatum and prefrontal cortex upon pharmacological interventions. Drug dose-effect relations are shown for GABAergic and glutamatergic interventions with vigabatrin, 3mercaptopropionate (3-MP), tiagabine, L-methionine sulfoximine (MSO), and riluzole, respectively. Data are represented as mean \pm SEM across 6-8 animals per group. Significance levels are indicated by asterisks for single doses tested against vehicle and for linear-trend tests across dose groups (arrows, corrected for multiple testing): (*)trend at $p<0.1$, ${ }^{*} p<0.05$, *** $p<0.01$ and ${ }^{*} * * * 0.001$. Dashed arrows denote a linear trend at $p<0.1$.

glutamine of $\sim 30 \%(p<0.05)$ in the striatum and $\sim 50 \%$ $(p<0.001)$ in the prefrontal cortex.

Finally, riluzole, a marketed drug with glutamatergic polypharmacology, was used to down-modulate presynaptic vesicular release and to enhance glial scavenging of glutamate. Glutamate was significantly and dose-dependently reduced in the striatum $(p<0.05$; corrected for multiple testing). In the prefrontal cortex this dose-effect was 
somewhat less pronounced but resulted in a highly significant reduction of glutamate by $\sim 7 \%(p<0.05$ and $p<0.01)$ at the higher doses. Glutamine levels remained unchanged upon intervention with riluzole

\section{Ex vivo Quantification of Drug Exposure and GABA and Glutamate Levels}

The findings obtained by in vivo pharmacoMRS were further validated with ex vivo LC-MS/MS analyses of GABA and glutamate levels and drug exposures in tissue samples harvested from the same animals. All pharmacological interventions tested (except 3-MP, which was not assessed) showed nearly dose-proportional exposures in blood and brain tissue and reached the expected tissue levels (see Supplementary Material).

Table 1 compares the outcomes of pharmacoMRS and LC-MS/MS measurements of cerebral GABA and glutamate concentrations in the animals of the vigabatrin arm. LCMS/MS qualitatively corroborated the brain region-related and drug-induced differences in neurometabolite levels observed in vivo by pharmacoMRS. GABA and glutamate concentrations reported by LC-MS/MS however were systematically higher by a factor of $\sim 1.2$ and $\sim 1.3$, respectively, when assuming a density of $1.044 \mathrm{~g} / \mathrm{ml}$ for brain tissue (DiResta et al, 1990).

\section{DISCUSSION}

The goal of the present study was to ascertain the value and limitations of in vivo ${ }^{1} \mathrm{H}$ parmacoMRS as a quantitative modality for noninvasively reporting levels of neurometabolites implicated in GABAergic and glutamatergic neurotransmission as putative biomarkers of state and

Table I Comparison of In vivo ' $H$ pharmacoMRS and Ex vivo LC-MS/MS Readouts of GABA and Glutamate Levels in Striatum and Prefrontal Cortex of Sprague-Dawley Rats Subjected to Pharmacological Interventions with Vigabatrin

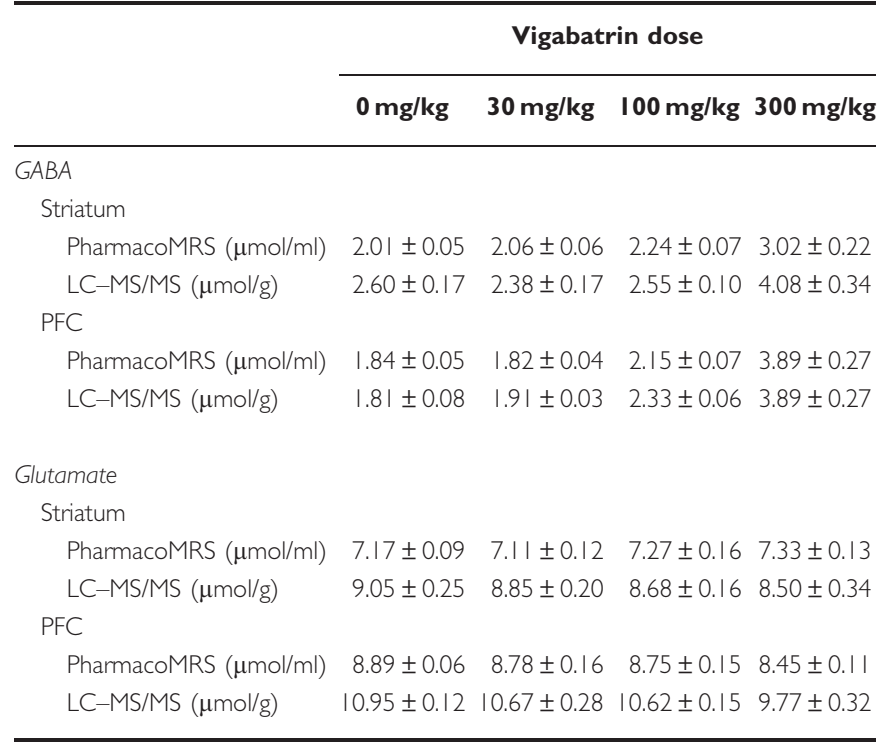

Data are presented as mean \pm SEM. pharmacodynamics in preclinical drug discovery in mental disorders.

\section{Quality, Reliability, and Generic Sensitivity under Routine Operation}

Preclinical drug discovery environments are characterized by extensive routine operation which stands up for standardization, reproducibility, and high throughput across all steps of the experimental procedures. For pharmacoMRS to be of added value in these settings, it has to seamlessly integrate and provide sensitive, quantitative, and relevant readouts. Here, the experimental duration was limited to less than $1 \mathrm{~h}$ per animal to mellow the potential impact on physiology as well as to increase throughput. Quantification of the spectral data was based on standard LCModel analysis that was fully automated to avoid user bias. All spectra and their LCModel fits obtained under these conditions of routine operation were of sustained high quality. Water line widths were at the propitious lower end of the $8-15 \mathrm{~Hz}$ range reported previously (Mlynarik et al, 2008; Tkac et al, 2003). The normalized SNR (normalized to volume and number of transients acquired but uncorrected for field strength) in the striatum and the cortex reached favorable values of 32 $\left(\mathrm{cm}^{-3} \times\right.$ transient $\left.^{-1 / 2}\right)$ and $43\left(\mathrm{~cm}^{-3} \times\right.$ transient $\left.^{-1 / 2}\right)$, thus approaching the SNR of $\sim 42\left(\mathrm{~cm}^{-3} \times\right.$ transient $\left.{ }^{-1 / 2}\right)$ reported for ${ }^{1} \mathrm{H}$-MRS in rat brain at the higher field strength of 16.4 T (Hong et al, 2011b). CRLBs for GABA, glutamate, and glutamine quantification were at the favorable very low end of the few values disclosed in previous preclinical studies (Cudalbu et al, 2009; Hong et al, 2011a; Pfeuffer et al, 1999; Tkac et al, 2004). At study level, variance components analysis on our data evidenced outstanding technical long-term stability over many months and remarkable biological consistency across different batches of rats. It is of further note that overall variability of GABA, glutamate, and glutamine measurements were on par with CRLBs, thus providing further support for well-balanced contributions of biological and technical variability. Taken together, spectral quality and reliability achieved here in routine investigations translate to propitious sensitivity limits for pharmacoMRS of GABA, glutamate, and glutamine that lie well within the range of tissular alterations reported for psychiatric disorders and respective pharmacological treatments in humans (Blum and Mann, 2002; Hashimoto et al, 2007).

\section{Neuropharmacological Relevance}

We further assessed the neuropharmacological relevance of pharmacoMRS readouts by probing their fidelity and sensitivity of reporting changes in neurometabolite levels in response to pharmacological interventions specifically targeting GABAergic and glutamatergic neurotransmission. Inhibition of glial GABA catabolism by vigabatrin has been shown by biochemical analysis of tissue, microdialysis, and MRS to substantially increase extracellular as well as intracellular levels of cerebral GABA (Errante and Petroff, 2003; Preece et al, 1994). Conversely, inhibition of GABA synthesis by 3-MP has been reported by biochemical procedures and microdialysis to lead to a reduction of 
tissular- and extracellular-GABA levels (Adcock and Taberner, 1978; Karlsson et al, 1974). Tiagabine is expected to have the least pronounced effect of the three GABAergic interventions tested. Its mode-of-action, which is based on the specific inhibition of presynaptic-GABA reuptake through GAT1 without affecting GABA scavenging by glia (GAT2/3), suggests that tiagabine increases primarily the local extracellular, presumably intrasynaptic levels of GABA. In fact, microdialysis studies revealed an up to fourfold increase in extracellular GABA after tiagabine administration (Fink-Jensen et al, 1992; Richards and Bowery, 1996) whereas biochemical analyses of cortices of mice subchronically treated with tiagabine reported the tissular-GABA pool to be unaffected (Leach et al, 1997). It is of particular note that pharmacoMRS, although it is thought to report predominantly on tissular neurometabolites, detected a small but evident increase in the striatal GABA. This observation suggests that the sensitivity of pharmacoMRS is sufficient to reveal relevant neurochemical changes in subcompartments and that assessments are not limited to pharmacological interventions with gross effects on bulk concentrations.

On the glutamatergic side, inhibition of the glutamine synthetase by MSO is known to result in a pronounced reduction of cerebral glutamate (Fonnum and Paulsen, 1990; Ghoddoussi et al, 2010). Likewise, riluzole is supposed to reduce neural glutamate levels by its polypharmacological action as suppressor of presynaptic vesicular glutamate release and enhancer of EAAT-mediated glutamate scavenging that together convey anticonvulsant and neuroprotective therapy (Banasr et al, 2010; Kim et al, 2007). Hence, our pharmacoMRS assessments have indeed faithfully reported these anticipated primary pharmacological effects of the latter prototypical GABAergic and glutamatergic interventions.

Our data also bolster the neuropharmacological relevance of pharmacoMRS from a quantitative stance. PharmacoMRS reported clear drug dose and exposure-dependent effects on GABA and glutamate over dose ranges that are pharmacologically sensible and have been shown to be behaviorally active in preclinical settings (Nielsen et al, 1991; Vinogradova et al, 2005). The experimental pro-convulsive drugs 3-MP and MSO were dosed according to precedent preclinical investigations and framed the respective seizure thresholds for nonanaesthetised rats at 30 (Lamar, 1970) and $150 \mathrm{mg} / \mathrm{kg}$ (Gershoff and Elvehjem, 1951), respectively. For the clinically approved drugs dose equivalents were compared on a $\mathrm{mg} / \mathrm{m}^{2}$ basis. Vigabatrin doses thus covered a $0.1-1.0$ fold equivalent of the maximum recommended human dose of $3000 \mathrm{mg} /$ day (Drugs at FDA, http:// www.accessdata.fda.gov/scripts/cder/drugsatfda/index.cfm). Tiagabine was administered at doses typically used in rats (Fink-Jensen et al, 1992; Richards and Bowery, 1996), covering a $0.7-2.7$ fold equivalent of the recommended human dose of $30 \mathrm{mg} /$ day for therapeutic sustenance (Drugs at FDA, http://www.accessdata.fda.gov/scripts/cder/ drugsatfda/index.cfm). Riluzole was given at $0.3-1.2$ fold equivalents of the recommended daily human dose of $2 \times 50 \mathrm{mg}$ (Drugs at FDA, http://www.accessdata.fda.gov/ scripts/cder/drugsatfda/index.cfm). Importantly, the minimal changes in GABA and glutamate levels reliably detected by pharmacoMRS were all elicited by drug doses well within the equivalent pharmacologically relevant dose range used in human studies (eg, (Petroff et al, 2000; Petroff and Rothman, 1998)), thus reaffirming that pharmacoMRS' sensitivity be suitable for investigating in vivo neuropharmacology in rats.

\section{Neurobiological Relevance}

The present data also underpins the neurobiological relevance of pharmacoMRS. Notably, pharmacoMRS revealed higher basal GABA and lower glutamate levels in the striatum than in the prefrontal cortex, thus confirming previous in vivo and ex vivo findings in drug naïve animals (Tkac et al, 2004; Tkac et al, 2003) and relating to the distinct neurofunctional features of the two entities. The striatum mainly comprises GABAergic medium spiny neurons whereas the prefrontal cortex has a predominantly glutamatergic innervation. Both areas are considered to be strongly implicated in the pathogenesis and etiology of numerous mental disorders. In this respect it is of particular note that the extent of GABA, glutamate, and glutamine responses to specific pharmacological interventions were found to be region-specific. For example, vigabatrin elicited a stronger change in GABA in the prefrontal cortex than in the striatum whereas for 3-MP and tiagabine this preference was reversed. Such region-specific responses are in concordance with previous studies (Halonen et al, 1991; Sheikh and Martin, 1998) and, although their basis is not fully explored, they may be rationalized by the intrinsic differential target expression, target occupancy, metabolic flux rates, and feedback regulation in the striatum and the prefrontal cortex. GABA-T and GAD65/67 have been reported to be more expressed in the striatum than in the prefrontal cortex whereas for GAD1 this preference is reversed (Durkin et al, 1995; Gale et al, 1984; Laprade and Soghomonian, 1999). Yet, forecasting directionality of correlations between expression levels and changes in metabolite pool sizes remains a challenge because fluxes through the pathways addressed by the respective pharmacological intervention were not directly amenable in the present study. In fact, changes in GABA levels observed after short-term treatment with GABA-T inhibitors have previously been used as a measure of GABAergic turnover. It also should be noted that isoflurane anesthesia used for the present in vivo assessments adds an inherent bias to the outcome in that it acts on $\mathrm{GABA}_{\mathrm{A}}$ receptors and other targets and thus affects the state of the neurotransmitter systems potentially also in a region-specific manner (Herring et al, 2009). Notwithstanding these anesthesiarelated effects, if tightly controlled by standardised conditions, do not curtail the outcome of the study.

GABAergic and glutamatergic neurotransmission are intricately intertwined both at the neurofunctional and neurochemical level (see Figure 3). Notably, pharmacoMRS unveiled such interactions. Vigabatrin and tiagabine with their explicit GABAergic modes-of-action elicited distinct downstream modulations of the glutamate pools in a region-specific and dose-dependent manner, thus further strengthening the neurobiological relevance of pharmacoMRS findings. In this respect, tiagabine may serve as an attractive example in that it revealed a modest and capped primary pharmacological effect on the striatal GABA pool whereas glutamate levels in the prefrontal cortex kept decreasing and the striatal glutamine steadily rose at 
increasing drug doses. For interventions with vigabatrin (Fonnum and Paulsen, 1990) and MSO (de Graaf et al, 1991; Ghoddoussi et al, 2010), previous studies reported decreased levels of glutamine that oppose our results. Particularly for $\mathrm{MSO}$, with its inhibitory activity on GS, in a linear system, accumulation of the substrate (glutamate) and depletion of the product (glutamine) would be expected. However, upstream and downstream effects in the multi-compartment system of the neuronal-glial glutamate/GABA-glutamine cycle with its feedback loops at the neurometabolic and neuroactivity tiers are more complex and underscore the value of comprehensive assessments. In this respect, pharmacoMRS in conjunction with ${ }^{13} \mathrm{C}$-labeled substrates as tracers may provide complementary information on neurometabolite trafficking as has been recently demonstrated by Chowdhury et al (Chowdhury et al, 2008) who reported increased glutamate-glutamine cycling at stable glutamate and glutamine levels in response to chronic riluzole treatment. The latter observation is in discordance with our finding of reduced glutamate levels after acute administration of riluzole. However, major differences between the two studies in treatment duration (repeated versus single dose), drug exposure (off drug versus acute exposure) and anesthesia may explain this apparent divergence.

Finally, additional corroborative support of the neurobiological and neuropharmacological findings gathered with in vivo pharmacoMRS was obtained from biochemical analyses with LC-MS/MS on tissue samples collected in the vigabatrin arm. Basal GABA and glutamate levels, and druginduced changes measured by both modalities ran parallel. On an absolute scale, however, LC-MS/MS systematically reported higher tissue concentrations than pharmacoMRS, thus deserving further discussion. For pharmacoMRS, referencing to tissue water was used for absolute scaling on the premise of a constant water concentration and with no correction for $\mathrm{T}_{1 \text { - and }} \mathrm{T}_{2}$-relaxation effects. Although this approach may have indeed introduced a bias, it avoids excessive variability to accrue from ancillary measurements required for scale adjustments. Additionally, it has been hypothesized that neural metabolites might be partially MRS invisible (Kauppinen and Williams, 1991). At the LCMS/MS end, the major blemish is tissue dehydration during the resection of small biopsies which leads to spurious and hardly controllable increases in metabolite concentrations. Most importantly, and in spite of the minor difference in absolute scaling, the present data imply that pharmacoMRS reliably reports localized changes in the pools of GABA and glutamate.

\section{CONCLUSION}

The current work has ascertained the value and limitations of quantitative pharmacoMRS for drug discovery efforts in preclinical research. In particular, sensitivity and fidelity of pharmacoMRS for noninvasively reporting changes in GABA and glutamate upon pharmacological interventions have been determined. Our data demonstrate that pharmacoMRS indeed provides neuropharmacologically and neurobiologically relevant readouts that can support decision making in preclinical research in mental disorders with the promise to be translational to clinical investigations.

\section{FUNDING AND DISCLOSURE}

$\mathrm{AB}, \mathrm{SM}, \mathrm{MK}, \mathrm{EB}, \mathrm{MvK}$, and $\mathrm{BK}$ have received salaries as compensation for their employment with F. Hoffmann-La Roche. MR has received salary as compensation for his employment with the ETH and University of Zürich. CFW was funded by the Roche Postdoctoral Fellowship program.

\section{ACKNOWLEDGEMENTS}

We gratefully acknowledge Stephanie Schöppenthau for skilled animal handling, Thomas Bielser for data processing, Véronique Stierlin for conducting the LC-MS/MS analyses, and Stephen Provencher for providing specific basis sets of model spectra for spectral fitting. This work was supported by the Roche Postdoctoral Fellowship Program.

\section{REFERENCES}

Adcock T, Taberner PV (1978). Measuring changes in cerebral glutamate and GABA metabolism prior to convulsions induced by 3-mercaptopropionate. Biochem Pharmacol 27: 246-248.

Banasr M, Chowdhury GM, Terwilliger R, Newton SS, Duman RS, Behar KL et al (2010). Glial pathology in an animal model of depression: reversal of stress-induced cellular, metabolic and behavioral deficits by the glutamate-modulating drug riluzole. Mol Psychiatry 15: 501-511.

Blum BP, Mann JJ (2002). The GABAergic system in schizophrenia. Int J Neuropsychopharmacol 5: 159-179.

Carlsson A, Waters N, Holm-Waters S, Tedroff J, Nilsson M, Carlsson ML (2001). Interactions between monoamines, glutamate, and GABA in schizophrenia: new evidence. Annu Rev Pharmacol Toxicol 41: 237-260.

Chowdhury GM, Banasr M, de Graaf RA, Rothman DL, Behar KL, Sanacora G (2008). Chronic riluzole treatment increases glucose metabolism in rat prefrontal cortex and hippocampus. J Cereb Blood Flow Metab 28: 1892-1897.

Cudalbu C, Mlynarik V, Xin L, Gruetter R (2009). Comparison of $\mathrm{T} 1$ relaxation times of the neurochemical profile in rat brain at 9.4 tesla and 14.1 tesla. Magn Reson Med 62: 862-867.

de Graaf AA, Deutz NE, Bosman DK, Chamuleau RA, de Haan JG, Bovee WM (1991). The use of in vivo proton NMR to study the effects of hyperammonemia in the rat cerebral cortex. NMR Biomed 4: 31-37.

de Graaf RA, Patel AB, Rothman DL, Behar KL (2006). Acute regulation of steady-state GABA levels following GABA-transaminase inhibition in rat cerebral cortex. Neurochem Int 48: 508-514.

DiResta GR, Lee J, Lau N, Ali F, Galicich JH, Arbit E (1990). Measurement of brain tissue density using pycnometry. Acta Neurochir Suppl (Wien) 51: 34-36.

Drugs at FDA. FDA approved drug products. http://www.accessdata. fda.gov/scripts/cder/drugsatfda/index.cfm, accessed 26 February 2014.

Durkin MM, Smith KE, Borden LA, Weinshank RL, Branchek TA, Gustafson EL (1995). Localization of messenger RNAs encoding three GABA transporters in rat brain: an in situ hybridization study. Brain Res Mol Brain Res 33: 7-21.

Errante LD, Petroff OA (2003). Acute effects of gabapentin and pregabalin on rat forebrain cellular GABA, glutamate, and glutamine concentrations. Seizure 12: 300-306.

Fink-Jensen A, Suzdak PD, Swedberg MD, Judge ME, Hansen L, Nielsen PG (1992). The gamma-aminobutyric acid (GABA) uptake inhibitor, tiagabine, increases extracellular brain levels of GABA in awake rats. Eur J Pharmacol 220: 197-201.

Fonnum F, Paulsen RE (1990). Comparison of transmitter amino acid levels in rat globus pallidus and neostriatum during 
hypoglycemia or after treatment with methionine sulfoximine or gamma-vinyl gamma-aminobutyric acid. J Neurochem 54: 1253-1257.

Gale K, Sarvey C, Stork J, Childs JA, Yalisove BL, Dayhoff RE (1984). Quantitative histochemical measurement of GABAtransaminase: method for evaluation of intracerebral lesions produced by excitotoxic agents. Brain Res 307: 255-262.

Gershoff SN, Elvehjem CA (1951). The relative effect of methionine sulfoximine on different animal species. J Nutr 45: 451-458.

Ghoddoussi F, Galloway MP, Jambekar A, Bame M, Needleman R, Brusilow WS (2010). Methionine sulfoximine, an inhibitor of glutamine synthetase, lowers brain glutamine and glutamate in a mouse model of ALS. J Neurol Sci 290: 41-47.

Halonen T, Pitkanen A, Saano V, Riekkinen PJ (1991). Effects of vigabatrin (gamma-vinyl GABA) on neurotransmission-related amino acids and on GABA and benzodiazepine receptor binding in rats. Epilepsia 32: 242-249.

Hashimoto K, Okamura N, Shimizu E, Iyo M (2004). Glutamate hypothesis of schizophrenia and approach for possible therapeutic drugs. Curr Med Chem 4: 147-154.

Hashimoto K, Sawa A, Iyo M (2007). Increased levels of glutamate in brains from patients with mood disorders. Biol Psychiatry 62: $1310-1316$.

Herring BE, Xie Z, Marks J, Fox AP (2009). Isoflurane inhibits the neurotransmitter release machinery. J Neurophysiol 102: 1265-1273.

Hong ST, Balla DZ, Pohmann R (2011a). Determination of regional variations and reproducibility in in vivo $1 \mathrm{H}$ NMR spectroscopy of the rat brain at 16.4 T. Magn Reson Med 66: 11-17.

Hong ST, Balla DZ, Shajan G, Choi C, Ugurbil K, Pohmann R (2011b). Enhanced neurochemical profile of the rat brain using in vivo (1) $\mathrm{H}$ NMR spectroscopy at 16.4 T. Magn Reson Med 65: 28-34.

Iltis I, Koski DM, Eberly LE, Nelson CD, Deelchand DK, Valette J et al (2009). Neurochemical changes in the rat prefrontal cortex following acute phencyclidine treatment: an in vivo localized (1)H MRS study. NMR Biomed 22: 737-744.

Karlsson A, Fonnum F, Malthe-Sorenssen D, Storm-Mathisen J (1974). Effect of the convulsive agent 3-mercaptopropionic acid on the levels of GABA, other amino acids and glutamate decarboxylase in different regions of the rat brain. Biochem Pharmacol 23: 3053-3061.

Kauppinen RA, Williams SR (1991). Nondestructive detection of glutamate by $1 \mathrm{H}$ nuclear magnetic resonance spectroscopy in cortical brain slices from the guinea pig: evidence for changes in detectability during severe anoxic insults. J Neurochem 57: 1136-1144.

Kim JE, Kim DS, Kwak SE, Choi HC, Song HK, Choi SY et al (2007). Anti-glutamatergic effect of riluzole: comparison with valproic acid. Neuroscience 147: 136-145.

Kim SY, Lee YJ, Kim H, Lee DW, Woo DC, Choi CB et al (2010). Desipramine attenuates forced swim test-induced behavioral and neurochemical alterations in mice: an in vivo(1)H-MRS study at 9.4T. Brain Res 1348: 105-113.

Krystal JH, Sanacora G, Blumberg H, Anand A, Charney DS, Marek $\mathrm{G}$ et al (2002). Glutamate and GABA systems as targets for novel antidepressant and mood-stabilizing treatments. Mol Psychiatry 7(Suppl 1): S71-S80.

Lamar C Jr. (1970). Mercaptopropionic acid: a convulsant that inhibits glutamate decarboxylase. J Neurochem 17: 165-170.

Laprade N, Soghomonian JJ (1999). Gene expression of the GAD67 and GAD65 isoforms of glutamate decarboxylase is differentially altered in subpopulations of striatal neurons in adult rats lesioned with 6-OHDA as neonates. Synapse 33: 36-48.

Leach JP, Sills GJ, Butler E, Forrest G, Thompson GG, Brodie MJ (1997). Neurochemical actions of vigabatrin and tiagabine alone and in combination in mouse cortex. Gen Pharmacol 28: 715-719.

Lee MR, Denic A, Hinton DJ, Mishra PK, Choi DS, Pirko I et al (2012). Preclinical (1)H-MRS neurochemical profiling in neurological and psychiatric disorders. Bioanalysis 4: 1787-1804.

Mattson RH, Scheyer RD, Petroff OA, During MJ, Collins TL, Spencer D (1998). Novel methods for studying new antiepileptic drug pharmacology. Adv Neurol 76: 105-112.

Mlynarik V, Kohler I, Gambarota G, Vaslin A, Clarke PGH, Gruetter R (2008). Quantitative proton spectroscopic imaging of the neurochemical profile in rat brain with microliter resolution at ultra-short echo times. Magn Reson Med 59: 52-58.

Moghaddam B, Javitt D (2012). From revolution to evolution: the glutamate hypothesis of schizophrenia and its implication for treatment. Neuropsychopharmacol 37: 4-15.

Nielsen EB, Suzdak PD, Andersen KE, Knutsen LJ, Sonnewald U, Braestrup C (1991). Characterization of tiagabine (NO-328), a new potent and selective GABA uptake inhibitor. Eur $J$ Pharmacol 196: 257-266.

Petroff O, Mattson RH, Rothman D (2000). Proton MRS: GABA and Glutamate. In: Henry TRDuncan JSBerkovic SF (eds) Functional Imaging in the Epilepsies. Lippincott Williams \& Wilkins: Philadelphia, pp 263-273.

Petroff OA, Rothman DL (1998). Measuring human brain GABA in vivo: effects of GABA-transaminase inhibition with vigabatrin. Mol Neurobiol 16: 97-121.

Pfeuffer J, Tkac I, Provencher SW, Gruetter R (1999). Toward an in vivo neurochemical profile: quantification of 18 metabolites in short-echo-time (1)H NMR spectra of the rat brain. J Magn Reson 141: 104-120.

Pierard C, Satabin P, Lagarde D, Barrere B, Guezennec CY, Menu JP et al (1995). Effects of a vigilance-enhancing drug, modafinil, on rat brain metabolism: a 2D COSY 1H-NMR study. Brain Res 693: $251-256$

Preece NE, Jackson GD, Houseman JA, Duncan JS, Williams SR (1994). Nuclear magnetic resonance detection of increased cortical GABA in vigabatrin-treated rats in vivo. Epilepsia 35: 431-436.

Provencher SW (1993). Estimation of metabolite concentrations from localized in vivo proton NMR spectra. Magn Reson Med 30: 672-679.

Richards DA, Bowery NG (1996). Comparative effects of the GABA uptake inhibitors, tiagabine and NNC-711, on extracellular GABA levels in the rat ventrolateral thalamus. Neurochem Res 21: $135-140$.

Sanacora G, Saricicek A (2007). GABAergic contributions to the pathophysiology of depression and the mechanism of antidepressant action. CNS Neurol Disord Drug Targets 6: 127-140.

Sheikh SN, Martin DL (1998). Elevation of brain GABA levels with vigabatrin (gamma-vinylGABA) differentially affects GAD65 and GAD67 expression in various regions of rat brain. J Neurosci Res 52: 736-741.

Tkac I, Henry PG, Andersen P, Keene CD, Low WC, Gruetter R (2004). Highly resolved in vivo $1 \mathrm{H}$ NMR spectroscopy of the mouse brain at 9.4 T. Magn Reson Med 52: 478-484.

Tkac I, Rao R, Georgieff MK, Gruetter R (2003). Developmental and regional changes in the neurochemical profile of the rat brain determined by in vivo $1 \mathrm{H}$ NMR spectroscopy. Magn Reson Med 50: 24-32.

Vinogradova LV, Kuznetsova GD, Shatskova AB, van Rijn CM (2005). Vigabatrin in low doses selectively suppresses the clonic component of audiogenically kindled seizures in rats. Epilepsia 46: $800-810$

Supplementary Information accompanies the paper on the Neuropsychopharmacology website (http://www.nature.com/npp) 